\title{
Monitoramento de interações farmacocinéticas entre plantas medicinais e fitoterápicos e os medicamentos convencionais pelo sistema de farmacovigilância brasileiro
}

\author{
Pharmacovigilance in Brazil: the monitoring of \\ herb-drug interactions using pharmacokinetic data
}

André Luís Dias Araujo MAZZARI, Jose Maria PRIETO

Department of Pharmaceutical \& Biological Chemistry, UCL School of Pharmacy, 29-39 Brunswick Square, WC1N1AX,London, UK. Email: andre.mazzari.12@ucl.ac.uk

\begin{abstract}
Consumption of herbal medicines has been increasing worldwide. In Brazil, the use of herbal medicines is expanding due to the misconception of the "innocuous nature" of this type of therapy. Herbal medicines, as like any other xenobiotic, may cause pharmacokinetic alterations in the organism, leading to changes in both phase 1 and phase 2 metabolism, due to enzyme inhibition or induction. In cases of the co-administration of herbal medicines with conventional drugs, the efficacy of the latter can be compromised, resulting in therapeutic failure. Because pharmacokinetic preclinical tests in herbal medicines are not a regulatory requirement, cases of herb-drug interactions (HDI) are mostly revealed in the post-market phase, i.e., by the pharmacovigilance system. Numerous countries have already reported cases of HDI through their respective pharmacovigilance systems to the World Health Organization (WHO), but Brazil did not have officially reported anyone so far. Moreover, during the first 10 years of monitoring, no HDI have been clearly established for Brazilian herbal medicines. It is known that, approximately, $90 \%$ of the Brazilian population use herbal medicines and, therefore, cases of HDI might be occurring without being noticed or formally reported. This could be changed by the adoption of several strategies, such as the continued education of health professionals, the encouragement of these professionals to report cases of HDI to the pharmacovigilance systems, the inclusion of traditional practitioners to the public health system and campaigns to prevent the self-medication of herbal medicines by the general public.
\end{abstract}

Key Words: Pharmacovigilance, Herb-Drug Interactions, Herbal Medicines, Medicinal Plants, Metabolism; Brazilian Health Surveillance Agency (ANVISA)

\section{RESUMO}

O consumo de plantas medicinais e fitoterápicos está cada vez mais em expansão pelo mundo. Contudo, os fitoterápicos, como qualquer xenobiótico, podem causar alterações farmacocinéticas no organismo, ocasionando distúrbios nos metabolismos de fase 1 e fase 2, devido à inibição ou indução de enzimas metabólicas. Em caso de coadministração de plantas medicinais e/ou fitoterápicos com medicamentos convencionais, poderá haver interações que comprometem a eficácia do último, ocasionando uma falha terapêutica. Como ensaios pré-clínicos farmacocinéticos não são uma exigência regulatória para que um medicamento fitoterápico seja registrado, os casos de interações com fármacos convencionais (Herb-Drug Interactions - HDI) acabam sendo revelados somente na fase de pós-comercialização, ou seja, pelos sistemas de farmacovigilância. Inúmeros países já reportaram casos de HDI através de seus respectivos centros de farmacovigilância à Organização Mundial de Saúde (OMS), mas até o momento, o Brasil não reportou nenhum oficialmente. Além disso, durante os primeiros 10 anos de monitoramento, nenhum caso de HDI envolvendo plantas medicinais brasileiras foi apresentado. Sabe-se que aproximadamente $90 \%$ da população brasileira fazem uso de plantas medicinais e fitoterápicos, e que casos de HDI podem estar acontecendo sem serem percebidos e/ou notificados. Para que este cenário mude, é necessário que se adote medidas que visem ao estímulo do profissional de saúde à comunicação de suspeita de HDI para o centro de farmacovigilância, bem como a educação continuada destes profissionais, com a inclusão de especialistas em medicina tradicional no sistema de saúde, além de campanhas de prevenção à automedicação de plantas medicinais e fitoterápicos pela população, que atualmente não é praticado no Brasil.

Palavras Chave: Farmacovigilância; Interações de medicamentos; medicamentos fitoterápicos; plantas medicinais; metabolismo; Agência Nacional de Vigilância Sanitária (ANVISA) 


\section{INTRODUÇÃO}

O consumo de plantas medicinais e fitoterápicos vem crescendo copiosamente no mundo, e em especial no Brasil. De acordo com dados da Organização Mundial de Saúde (OMS), cerca de $80 \%$ da população que vive em países em desenvolvimento, dependem exclusivamente da medicina tradicional, incluindo plantas medicinais para o tratamento de diversas doenças (1). No Brasil, os números apontam que mais de $90 \%$ dos brasileiros já fizeram uso de plantas medicinais, mostrando o grande potencial deste mercado no país (2). Este grande percentual de uso de plantas medicinais e fitoterápicos no Brasil pode ser justificado por algumas razões, como por exemplo: alto custo de medicamentos convencionais, grande disponibilidade no mercado, ou até mesmo pelo fato do medicamento fitoterápico ser considerado mais "seguro" e "sem efeitos colaterais" pela população (3).

Desde o estabelecimento da Política Nacional de Práticas Integrativas e Complementares (PNPIC) em 2006, o Ministério da Saúde (MS) vem ampliando o acesso seguro às plantas medicinais e fitoterápicos para a população por meio de sua implementação no Sistema Público de Saúde (SUS) (4). Além da PNPIC, no mesmo ano, o MS também elaborou a Política Nacional de Plantas Medicinais e Fitoterápicos (PNPMF), com o objetivo maior de promover o uso racional de fitoterápicos no Brasil por meio de diretrizes que regulamentam desde o cultivo das plantas medicinais até a produção e comercialização do fitoterápicos por indústrias farmacêuticas nacionais (5). Entretanto, muitos destes produtos são comercializados em feiras livres, mercados populares e estabelecimentos comerciais, sendo na maioria das vezes produzidos e consumidos inadequadamente, podendo causar, portanto, riscos à saúde dos pacientes (6).

Além dos perigos relacionados à qualidade do fitoterápico, um importante fator deve ser considerado ao se consumir este tipo de produto: a sua interação com medicamentos convencionais. Casos de reações adversas a medicamentos (RAM) ocasionadas por interações de plantas medicinais e/ou fitoterápicos com fármacos convencionais (HDIs) são comuns e podem ocasionar problemas sérios à saúde em decorrência de falha terapêutica. As plantas medicinais e fitoterápicos, quando consumidos, podem causar distúrbios farmacodinâmicos, produzindo aumento ou diminuição do(s) efeito(s) farmacológico(s), e farmacocinéticos, ocasionando problemas relacionados à absorção, distribuição, metabolização e excreção (ADME) de medicamentos convencionais, caso estes sejam coadministrados, devido à indução ou inibição de enzimas metabolizadoras (7).

Grandes variedades de espécies vegetais com atividade farmacológica reconhecida por meio de testes em laboratório ou até mesmo por uso tradicional estão disponíveis no mercado brasileiro. Contudo, pouco se conhece sobre os efeitos farmacocinéticos que estas plantas medicinais podem causar no indivíduo. Isto faz com que as interações farmacocinéticas sejam mais difíceis de serem previstas ou serem detectadas, quando comparadas com as interações farmacodinâmicas. Por exemplo, é mais simples verificar se a coadministração de fitoterápicos e medicamentos convencionais com atividade hipoglicemiante pode aumentar o risco de choque hipoglicêmico no paciente, do que averiguar se os mesmos podem causar alguma interação em nível da ADME (8).

Para evitar que estes efeitos indesejáveis e prejudiciais afetem a vida dos pacientes, as HDI devem ser cuidadosamente avaliadas pelos métodos de farmacologia pré-clínica e clínica e, principalmente, pelo sistema de farmacovigilância (caso exista) do país em questão (7). A necessidade de desenvolver um programa de vigilância de medicamentos tornou-se prioridade para a OMS após a tragédia da talidomida, em 1961. Sistemas de monitoramento de notificações espontâneas adversas foram implementados inicialmente em países como Austrália, Canadá, Estados Unidos, Irlanda, Japão, Nova Zelândia, Alemanha e Reino Unido (9). No Brasil, o registro e a fiscalização de medicamentos acontecem desde a época do Brasil Colônia. Porém, pode ser observada uma maior estruturação quanto à vigilância destes produtos na década de 70, devido aos crescentes casos de malformação congênita, envenenamentos na infância, abortos, etc (10). Após sua fundação, em 1999, a Agência Nacional de Vigilância Sanitária (Anvisa) instituiu o sistema de farmacovigilância em 2001 com a criação do Centro Nacional de Monitorização de Medicamentos (CNMM), que aprimorou-se ao longo dos anos e que, em 2009, culminou com a criação do Sistema Nacional de notificações para a Vigilância Sanitária (VIGIPÓS/Notivisa) (8).

Mesmo com um sistema de farmacovigilância em conformidade com o estabelecido pela Organização Mundial de Saúde (OMS), o Brasil ainda possui dificuldades em obter e divulgar dados referentes à HDI, que desencadeiam, portanto, preocupações quanto aos riscos associados à utilização de plantas medicinais e fitoterápicos em conjunto com medicamentos convencionais.

O objetivo desta nota técnica é mostrar como o Brasil se posiciona perante os demais países do mundo em relação à publicação de dados referentes à HDI oriundos do sistema nacional de farmacovigilância, e também mostrar como as plantas medicinais e fitoterápicos atualmente disponibilizadas no SUS podem ser causadoras de interações farmacocinéticas com medicamentos convencionais. 
Regulamentação de medicamentos fitoterápicos no Brasil

Atualmente, a Anvisa regula a produção e o comércio de fitoterápicos no Brasil por meio de normas como a Resolução da Diretoria Colegiada (RDC) 26/14 e a Instrução Normativa (IN) 02/14. A Relação Nacional de Medicamentos Essenciais (RENAME), também apresenta uma lista com fitoterápicos para atender às necessidades dos usuários do SUS (11-13). Uma nova lista de espécies vegetais que possuem atividade farmacológica poderá integrar o grupo de fitoterápicos oferecidos pelo SUS em breve. A Relação Nacional de Plantas Medicinais de Interesse ao SUS (RENISUS), lançada oficialmente em 2009 pelo Ministério da Saúde, traz uma lista com 71 espécies de plantas medicinais que, após terem sua eficácia e segurança comprovadas, poderão ser novas alternativas para o tratamento de determinadas doenças (14).

Apesar das regulamentações supracitadas minimizarem os riscos que as plantas medicinais e fitoterápicos podem provocar à saúde, pouco se sabe das implicações farmacocinéticas que estes xenobióticos podem causar ao organismo. Estudos pré-clínicos farmacocinéticos em fitoterápicos não são requeridos pelo sistema regulatório para que os mesmos sejam aprovados pela Anvisa. Consequentemente, as chances de HDI podem ser bem maiores do que o esperado (15).

\section{Dados de interações entre as plantas medicinais e fitoterápicos e os medicamentos convencionais no Brasil e no mundo}

Após a publicação do International Drug Monitoring Programme pela OMS, os centros nacionais de farmacovigilância dos países membros da Organização ficaram responsáveis por coletar, processar e analisar os casos suspeitos de reações adversas oriundas de interações entre plantas medicinais e fitoterápicos e medicamentos convencionais. Um artigo publicado em 2012 revelou, por meio de dados oficiais coletados no VigiSearch (ferramenta pela qual a OMS armazena os casos de RAM recebidos pelos centros de farmacovigilância de países membros), que 811 casos de HDI foram recebidos pelo sistema, o que corresponde a somente $4,6 \%$ do total de casos reportados em relação às plantas medicinais e fitoterápicos, e $0,012 \%$ em relação ao número total, incluindo medicamentos convencionais. Destaca-se também a ausência do Brasil como país onde casos de HDI foram recebidos pelo sistema VigiSearch (7). Contudo, a ausência destes dados não significa que HDI não ocorrem no país. Por exemplo, nosso recente estudo apontou que cerca de $40 \%$ das espécies vegetais da RENISUS, que possuem dados farmacocinéticos conhecidos, podem causar interações farmacocinéticas que interferem diretamente com as enzimas metabólicas, responsáveis pelo metabolismo de fase 1 e fase 2, além de alterarem a expressão da glicoproteína $\mathrm{P}$, que por sua vez, resulta em problemas de absorção dos medicamentos (8). Para fitoterápicos presentes na lista de registro simplificado (IN 02/14) e na RENAME, como Allium sativum, Glycine max, Mentha x piperita e Zingiber offcinale também já existem dados farmacocinéticos publicados, indicando como os mesmos podem interferir com o metabolismo e causar reações adversas decorrentes de interações com medicamentos convencionais (1619). Portanto, é possível prever que cerca da metade das plantas medicinais da RENISUS sem dados farmacocinéticos publicados podem, de alguma forma, interagir em algum processo da ADME.

\section{A farmacovigilância e possíveis razões para a ausência de dados sobre HDI no Brasil}

Atualmente, o CNMM utiliza a plataforma eletrônica NOTIVISA para a coleta de dados de RAM pelo país. O NOTIVISA foi criado em 2009 para receber notificações de eventos adversos (EA) e queixas técnicas (QT) de diversos produtos sob vigilância sanitária, incluindo medicamentos fitoterápicos. Por este sistema, estas informações são recebidas por profissionais de serviços de saúde, como hospitais, clínicas e laboratórios, pela Anvisa e vigilância sanitárias locais, além de profissionais que atuam em drogarias e farmácias. O sistema NOTIVISA permite também que cidadãos notifiquem qualquer caso de EA e QT, possibilitando um maior fornecimento de dados relacionados à segurança do medicamento (8).

Um estudo publicado em 2010 revelou que 165 EA a fitoterápicos foram recebidas pelo centro de farmacovigilância da Anvisa entre 1999 e 2009. Dentre eles, somente dois podem ter ocorrido em decorrência de HDI, ainda que estes casos não avaliem a probabilidade de tais causas: Atropa belladonna, administrada com acriflavina, metenamina e metiltionínio, causando hepatite no paciente, e o Pygeum africanum, que provocou cefaleia devido ao seu uso em conjunto com fumarato de rupatadina. O mesmo estudo também mostrou que $51 \%$ dos EA causados pelo consumo de plantas medicinais e fitoterápicos foram reportados por profissionais de saúde. Considerando que os profissionais de saúde são mais qualificados e, portanto, mais indicados para relatar RAM, este percentual encontra-se abaixo do esperado (20).

A carência de informações sobre HDI no país pode ter relação com alguns fatores, como a falta de promoção do uso racional de plantas medicinais e fitoterápicos e deficiência no treinamento de profissionais de saúde aptos a 
detectarem estes casos. Verifica-se hoje um número alto de profissionais que podem legalmente prescrever plantas medicinais e fitoterápicos para a população. Além dos médicos, os odontólogos, enfermeiros, farmacêuticos e nutricionistas possuem autorização de seus respectivos conselhos federais para a prática da prescrição destes produtos (21). Contudo, muitos destes profissionais não possuem em sua matriz curricular conteúdos com carga horária compatível que os habilitem a serem profissionais prescritores de plantas medicinais e drogas vegetais, dificultando ainda mais a detecção de HDI (22).

Pode-se também atribuir este resultado aos pacientes que fazem uso da automedicação. Devido à crença da "naturalidade inócua" das plantas medicinais e dos fitoterápicos, muitos pacientes não relatam seu uso aos médicos e outros profissionais de saúde, o que contribui, portanto, com a escassez de dados desta natureza (20).

\section{Regulamentação de profissionais especializados em medicina tradicional como potenciais colaboradores dos profissionais de saúde}

Devido à emergência do uso de plantas medicinais e fitoterápicos pelo mundo, a OMS iniciou um trabalho de formulação e implementação de estratégias para que estes produtos fossem utilizados de forma correta $\mathrm{e}$ segura pela população. Dentre as medidas produzidas, destaca-se a utilização de herbalistas, ou profissionais especializados em medicina tradicional (Traditional Medicine Practitioners, ou TMP em inglês) na saúde (23). Esta prática que vem sendo utilizada em países africanos, que dependem quase que exclusivamente de plantas medicinais como forma de tratamento para doenças, integrando os TMP nos sistemas locais de saúde para trabalharem em conjunto com os profissionais de saúde convencionais (Conventional Healthcare Practitioners, ou CHP em inglês). Para que esta medida fosse implementada, a OMS publicou, em 1995, um guia para o treinamento destes profissionais para sua inclusão nos sistemas de saúde locais. O TMP tem sua competência reconhecida pelas comunidades locais para diagnosticar e contribuir com o bem estar físico, mental, social e espiritual dos membros de suas respectivas comunidades (24). Os TMP também são treinados para detectar e tratar as doenças consideradas mais prevalentes em suas respectivas regiões, além de identificar reações adversas que podem ocorrer durante o tratamento. Em 2001, a OMS também emitiu o documento "Promoting the Role of Traditional Medicine in Health Systems: A Strategy for the African Region" promovendo a colaboração efetiva entre TMP e CHP inicialmente para o controle da AIDS no continente africano (25). Países como Mali, Senegal, Zâmbia, Uganda, Botswana, Malawi, Moçambique, África do Sul e República Centro-Africana já ob- servaram benefícios desta cooperação. Por exemplo, em Mali, a colaboração entre TMP e CHP resultou em um declínio na mortalidade causada por malária de 5\% em 1997 para $\%$ em 1998, além da redução da mortalidade pela forma severa da doença, que caiu de $38 \%$ para $10 \%$ em 2008 (26).

Na Europa, existe também um movimento para a regulamentação de profissionais em medicina tradicional no sistema de saúde. O MHRA (Medicines and Healthcare products Regulatory Agency), agência reguladora do Reino Unido, iniciou a formulação de propostas para o registro oficial destes profissionais, com o objetivo de contribuir com a segurança do uso de plantas medicinais e fitoterápicos no país (27).

No Brasil, a inclusão de TMP é destacada na PNPIC. A Diretriz PMF 5 da Política menciona a valorização dos detentores de conhecimento tradicional e sua participação na ampliação do conhecimento do uso tradicional de plantas medicinais. A PNPIC ainda aponta a necessidade da inclusão de TMP no SUS, que vai de encontro à necessidade de educação permanente dos profissionais de saúde na utilização de plantas medicinais e fitoterápicos, que também é enfatizada na Diretriz PMF 3 do documento (4).

Com esta mudança, espera-se que as plantas medicinais e fitoterápicos sejam utilizados de forma efetiva e racional, evitando também a sua coadministração com medicamentos convencionais para o tratamento de doenças e, portanto, auxiliando na prevenção de possíveis casos de HDI (27).

\section{Medidas para prevenção de HDIs}

Um importante passo para que casos de HDI sejam prevenidos ou mesmo reduzidos, é a conscientização não somente dos profissionais de saúde, mas também das autoridades regulatórias e consumidores em geral, dos problemas que as plantas medicinais e os fitoterápicos podem causar em caso de coadministração com medicamentos convencionais. Os crescentes casos de automedicação com estes produtos colocam em risco a saúde do paciente, principalmente quando não há acompanhamento médico. Portanto, profissionais de saúde em geral (médico, farmacêuticos, enfermeiros, etc) devem encorajar seus pacientes a informar-lhes sobre o uso de qualquer planta medicinal ou fitoterápico. Medidas como esta podem evitar, diretamente, possíveis casos de HDI. Em caso de suspeita de HDI, os profissionais de saúde devem reportá-los imediatamente ao serviço de farmacovigilância, para que a Anvisa publique um alerta sobre riscos de interações entre os medicamentos em questão, que serão também de uso da OMS (7). 


\section{CONCLUSÃO}

Devido ao constante aumento do consumo de plantas medicinais e fitoterápicos no Brasil, as chances de interações com medicamentos convencionais também são maiores. Aliando-se a este agravante, a não obrigatoriedade de estudos farmacocinéticos pré-clínicos em fitoterápicos para o registro desta classe de medicamentos contribui também para que casos de HDI ocorram.

Sabe-se que a difusão da fitoterapia no Brasil, orientada por políticas como o PNPIC e o PNPMF, vai de encontro aos anseios da OMS para que a biodiversidade do país seja utilizada em benefício da população. Contudo, como os efeitos farmacocinéticos de plantas medicinais tradicionalmente utilizadas no Brasil mostraram ser potenciais causadores de efeitos adversos por suas interações com medicamentos convencionais, medidas devem ser adotadas para que a população não sofra diretamente com os impactos provocados por estes acontecimentos.

Esforços para a determinação do perfil farmacocinético das plantas medicinais e fitoterápi- cos, envolvendo as universidades e os fornecedores destes materiais, devem ser promovidos através de programas do governo e de mudanças na regulamentação destes produtos. A promoção do uso racional de plantas medicinais e fitoterápicos pela população, bem como a educação continuada de profissionais de saúde nestas áreas, além da inclusão de TMP no sistema de saúde são aspectos importantes para que este tipo de terapia seja realizada com segurança Somando-se a estes fatos, o estímulo à informação de casos de HDI ao sistema de farmacovigilância e à OMS precisa ser praticado para que haja maior transparência em relação a este problema de saúde pública, que, no momento, mostra-se invisível.

\section{AGRADECIMENTOS}

Os autores agradecem o apoio financeiro da Ao Conselho Nacional de Desenvolvimento Científico e Tecnológico $(\mathrm{CNPq})$ e ao programa Ciência sem Fronteiras por financiarem o bolsista André Mazzari em seu doutorado pleno no exterior

\section{REFERÊNCIAS}

1. Organização Mundial da Saúde. New WHO guidelines to promote proper use of alternative medicines. Genebra, Suíça. 2004. Disponível em http://www.who.int/mediacentre/news/releases/2004/pr44/en/. [Acesso em 24 de agosto de 2014].

2. Corrêa CC, Alves AF. Plantas medicinais como alternativa de negócios: caracterização e importância. XLVI Congresso da Sociedade Brasileira de Economia, Administração e Sociologia Rural. 2008. Rio Branco - Acre. 2008.

3. Silveira PF, Bandeira MAM, Arrais PSD. Farmacovigilância e reações adversas às plantas medicinais e fitoterápicos: uma realidade. Revista Brasileira de Farmacognosia. 2008;18(4):618-26.

4. BRASIL. Ministério da Saúde. Política Nacional de Práticas Integrativas e Complementares no SUS Disponível em: http://189.28.128.100/dab/docs/publicacoes/geral/ pnpic.pdf [Acesso em 13 de fevereiro de 2014].

5. BRASIL. SUS. Política Nacional de Plantas Medicinais e Fitoterápicos. Brasília - DF - Brasil. 2006. Disponível em http://bvsms.saude.gov.br/bvs/publicacoes/politica_nacional_fitoterapicos.pdf [Acesso em 10 de fevereiro de 2014].

6. Santos RL, Guimaraes GP, Nobre MSC, Portela AS. Análise sobre a fitoterapia como prática integrativa no Sistema Único de Saúde. Revista Brasileira de Plantas Medicinais. 2011;13(4):486-91.
7. Skalli S, Soulaymani Bencheikh R. Safety monitoring of herb-drug interactions: a component of pharmacovigilance. Drug Saf. 2012;35(10):785-91.

8. Mazzari ALDA, Prieto JM. Herbal Medicines in Brazil: Pharmacokinetic Profile and Potential Herb-Drug Interactions. Frontiers in Pharmacology. 2014;5(162):1-12.

9. Rozenfeld S, Rangel ITM. A farmacovigilância. Cadernos de Saúde Pública. 1988;4(3):336-41.

10. Rozenfeld S. Farmacovigilância: elementos para a discussão e perspectivas. Cadernos de Saúde Pública. 1998;14(2):237-63.

11. BRASIL. SUS. Relação de Medicamentos Essenciais (RENAME) Disponível em: http://portal.saude.gov.br/ portal/arquivos/pdf/rename2010final.pdf Brasília - DF Brasil2010 [Acesso em 10 de junho de 2013].

12. BRASIL. Agência Nacional de Vigilância Sanitária. Instrução Normativa n 2, de 13 de maio de 2014. Publica a "lista de medicamentos fitoterápicos de registro simplificado" e a "lista de produtos tradicionais fitoterápicos de registro simplificado". (2014).

13. BRASIL. Agência Nacional de Vigilância Sanitária. Resolução RDC n 26, de 13 de maio de 2014. Dispõe sobre o registro de medicamentos fitoterápicos e o registro e a notificação de produtos tradicionais fitoterápicos., (2014). 
14. BRASIL. SUS. Relação Nacional de Plantas Medicinais de Interesse ao SUS (RENISUS). Disponível em http:// portal.saude.gov.br/portal/arquivos/pdf/RENISUS.pdf Brasilia - DF - Brasil2009 [Acesso em 3 de maio de 2013].

15. Hu Z, Yang X, Ho PC, Chan SY, Heng PW, Chan E, et al. Herb-drug interactions: a literature review. Drugs. 2005;65(9):1239-82.

16. Le Bon AM, Vernevaut MF, Guenot L, Kahane R, Auger J, Arnault I, et al. Effects of garlic powders with varying alliin contents on hepatic drug metabolizing enzymes in rats. J Agric Food Chem. 2003;51(26):7617-23.

17. Shon YH, Nam KS. Inhibition of cytochrome P450 isozymes and ornithine decarboxylase activities by polysaccharides from soybeans fermented with Phellinus igniarius or Agrocybe cylindracea. Biotechnol Lett. 2004;26(2):159-63.

18. Unger M, Frank A. Simultaneous determination of the inhibitory potency of herbal extracts on the activity of six major cytochrome P450 enzymes using liquid chromatography/mass spectrometry and automated online extraction. Rapid Commun Mass Spectrom. 2004;18(19):2273-81.

19. Ajith TA, Hema U, Aswathy MS. Zingiber officinale Roscoe prevents acetaminophen-induced acute hepatotoxicity by enhancing hepatic antioxidant status. Food Chem Toxicol. 2007;45(11):2267-72.

20. Balbino EE, Dias MF. Farmacovigilância: um passo em direção ao uso racional de plantas medicinais e fitoterápicos. Revista Brasileira de Farmacognosia. 2010;20:992-1000.
21. Camargo S, Barros de Leça Pereira V. A prática da fitoterapia pelo nutricionista - algumas reflexões. Revista da Associação Brasileira de Nutrição. 2013;5(1):69-72.

22. Rates SMK. Promoção do uso racional de fitoterápicos: uma abordagem no ensino de Farmacognosia. Revista Brasileira de Farmacognosia. 2001;11(2):57-69.

23. Amole O. The Role of Traditional Medicine in Primary Health Care. Journal of Immunodeficiency \& Disorders. 2012;1(1):1.

24. Santé Omdl. Traditional practitioners as primary health care workers: a study of effectiveness of four training projects in Ghana, Mexico and Bangladesh: Division of strengthening of health services and Traditional medicine programme; 1995.

25. Busia K, Kasilo O. Collaboration between traditional health practitioners and conventional health practitioners: Some country experiences. 2010. Disponível em http:// www.aho.afro.who.int/en/ahm/issue/13/reports/collaboration-between-traditional-health-practitioners-and-conventional-health. [Acesso em 04 de agosto de 2014].

26. King R, Homsy J. Involving traditional healers in AIDS education and counselling in sub-Saharan Africa: a review. Aids. 1997;11 Suppl A:S217-25.

27. MHRA. Herbal Medicines Regulation: Unlicensed herbal medicines supplied by a practitioner following a one-toone consultation. Disponível em: http://www.mhra.gov. uk/Howweregulate/Medicines/Herbalmedicinesregulation/Unlicensedherbalmedicinessuppliedbyapractitionerfollowingaonetooneconsultation/index.htm [Acesso em 15 de julho de 2014]. 